\title{
Enzymatic Glycosylations on Arrays
}

\author{
Josef Voglmeir, Robert Šardzík, Martin J. Weissenborn, and Sabine L. Flitsch
}

\begin{abstract}
The enzymatic glycosylation of microarrays is a relatively young field in glycoscience. Platforms developed from other array technologies (e.g., proteins and nucleic acids) were successfully adopted in several proof-of-principle studies as a high-throughput tool for the generation of more complex carbohydrate structures using carbohydrate-processing enzymes. These arrays and the developed on-chip enzymatic glycosylation methodologies are reviewed in this article.
\end{abstract}

\section{Introduction}

$\mathbf{U}$ NLIKE NUCLEIC ACIDS AND PROTEIN SEQUENCES, the Sequences of carbohydrates are not directly encoded in the genome, but are determined by the expression and specificity of a set of "glycoenzymes" such as glycosyltransferases and glycosidases, which are responsible for the biosynthesis and processing of carbohydrate structures. It has been estimated that around $2 \%$ of the genome is dedicated to such glycoenzymes. Given that such enzymes can have many (glyco)protein and glycolipid substrates, the impact of glycosylation is much larger; for example, it has been estimated that over $70 \%$ of all proteins in humans are glycosylated. Because each glycoenzyme has many different lipid and protein substrates, many of the "genetic" tools currently used in proteomics such as knock-out strains, iRNA, or two-hybrid systems are not as informative for glycomics as in the other "omic" disciplines. Most successful in the glycosciences have been biochemical and biophysical tools such as mass spectrometry for the determination of carbohydrate structures, enzymology of the glycoenzymes to determine their substrate specificity, and hence, predict glycan structures, and the applications of the lectins and glycoenzymes themselves as tools to generate and characterize glycan structures. Such biochemical studies have been slow and have given us very valuable yet patchy information of protein-carbohydrate interactions. In recent times, glycoscientists have therefore identified carbohydrate microarrays (glycoarrays) as the key tool for the high-throughput studies that are necessary to understand this complex area of biology.

The first generation of glycoarrays have been used to identify carbohydrate-protein binding interactions, and this application of glycoarrays has been reviewed elsewhere (Laurent et al., 2008b; Park et al., 2008). These studies have shown that carbohydrate arrays are particularly useful tools for glycomics: not only do they shorten the time of biological measurements, but they have also the advantage of using less quantities of precious carbohydrate material due to miniaturization that is not possible in solution studies. Although many advances have been made in recent years to isolate and synthesize oligosaccharides and glycoconjugates chemically or chemoenzymatically (Blixt and Razi, 2006; Davis, 2000; Nicolaou and Mitchell, 2001; Seeberger and Haase, 2000; Zhang et al., 1999), the quantities obtained are mostly in the low milligram scale, and therefore too small for extensive tests in conventional biochemical studies. The use of carbohydrate arrays to study carbohydrate-lectin/antibody binding has allowed to reduce the amount of immobilized carbohydrates to a femtogram scale, compared to the picogram scale required for ELISA-based screening or nanogram scale in immunodot assays (Willats et al., 2002).

The present review will focus on the application of glycoarrays to study enzymatic reactions involving Carbohydrate Active Enzymes, in particular, glycosyltransferases and glycosidases. These enzymes form a significant part of the proteome of higher organisms and their sequenced and biochemical data are well recorded on the Carbohydrate Active Enzymes database (http://www.cazy.org/) (Cantarel et al., 2009).

\section{Glycosylation Reactions on Array Platforms}

The specificity of enzymes involved in formation or hydrolysis of glycosidic linkages (Fig. 1) relates to three components of the glycosidic bond: specificity for the carbohydrate glycoside (glycosyl donor), stereospecificity for alpha or beta linkages and specificity for the aglycon component (glycosyl acceptor in the synthesis direction). Although genes corresponding to glycoenzymes can be identified in genomic databases based on consensus sequences and homology to known enzymes, the bioinformatic tools for predictions of selectivity of a given enzyme are currently not accurate, and

Manchester Interdisciplinary Biocentre, University of Manchester, Manchester M1 7ND, UK. 


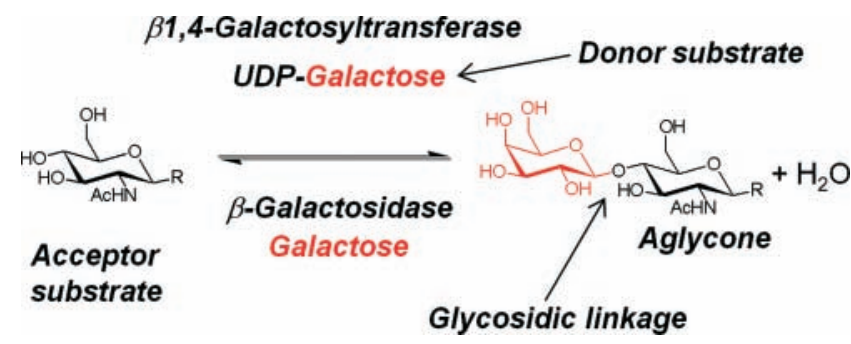

FIG. 1. Substrate specificity of glycoenzymes for acceptor, donor, and glycosidic linkage using galactosylation as an example.

biochemical data for substrate specificity are required. Given the complexity of substrates for such enzymatic reactions and the number of enzymes that need to be investigated, highthroughput array platforms are considered key tools for the determination of substrate specificity.

Array experiments involve the preparation of defined carbohydrate arrays that can be interrogated by a glycosyltransferase for acceptor specificity in the presence of defined glycosyl donors or be examined as a substrate for glycosidases. Table 1 presents a list of enzymatic reactions that have been studied so far. This list shows that a diversity of enzymes and array platforms have been used.

Except for the glycosyltransferase R (GTFR) used in the work of Seibel et al. (2006), which is a non-Leloir-type glucosyltransferase from Streptococcus oralis, all listed glycosyltransferases have used nucleotide sugars as activated donor substrates. Array technologies described are diverse and have generally been adopted from other array platforms, including glass slides, microtiter plates, and self-assembled monolayers (SAMs) on gold. The choice of readout technologies is also diverse and presents a challenge in glycosylation reactions, because carbohydrates do not generally contain chromophores. In the following we will discuss glycan arrays according to three general detection methods used: detection of glycosylation by using labeled glycosyl donors, by using lectins and antibodies for product detection, and label-free methods using mass spectrometry.

\section{Detection of Glycosylation Reactions Using Labeled Glycosyl Donors}

Figure 2 outlines the general strategy of using labeled glycosyl donors to monitor glycosylating enzymes. Blixt et al. (2008b) have utilized array technology to evaluate the substrate specificity of various sialyltransferases (Fig. 3). A glycan platform based on glass slides containing more than 200 potential sialyltransferase acceptor substrates was incubated with recombinant mammalian sialyltransferases and CMPNeu5Ac as donor substrate, which was biotinylated at the position 9 of the Neu5Ac (CMP-9Biot-Neu5Ac). The transfer of the biotinylated sialic acids onto the microarray could be detected using fluorescein labeled streptavidin. Each enzyme showed a specific glycosylation profile, and in several cases new specificities could be revealed. For example, it was demonstrated that the human $\alpha 2,6$-sialyltransferase I (hST6Gal-I) has the ability to accept structures terminating with GlcNAc $\beta 1,4 \mathrm{GlcNAc}$. The rat $\alpha 2,3$-sialyltransferase (rST3Gal-III) also allows fucosylated acceptors such as Lewis ${ }^{a}$, human $\alpha 2,3$-sialyltransferase IV (hST3Gal-IV) had a broad acceptor specificity for Gal $\beta 1,3 / 4 \mathrm{GlcNAc}$ and Gal $\beta 1,3 \mathrm{GalNAc}$ structures, and porcine $\alpha 2,3$-sialyltransferase I (pST3Gal-I) also accepted ganglio-oligosaccharides and core- 2 structures.

Faik and coworkers (Shipp et al., 2008) have generated a plant cell wall glycoarray platform for analyzing both enzymes as well as acceptors involved in cell wall biosynthesis. Their work has focused on fucosyltransferase 1 (AtFUT1). Chemically derivatized cello-oligosaccharides were attached onto photoactivatable glass slides. Using $\left[{ }^{14} \mathrm{C}\right] \mathrm{GDP}-\mathrm{Fuc}$ as a donor substrate, fucosylation of the immobilized acceptor was detected with a phosphoimager scanner. Interestingly, the fucosylation of some of the smaller acceptors, such of the xyloglucan trimer, strongly depends on the anchoring on the array surface. Experiments showed that no fucosylation was detected when the trimer was randomly immobilized on the chip without covalent attachment.

Chevolot et al. (2001) investigated the application of galactose and lactose-modified polystyrene (PS) surfaces. Their immobilization technology is based on a photochemical carbene formation and a subsequent reaction with the polymeric solid support (Fig. 4). Lactose modified with a linker containing a light sensitive aryl diazirine group was prepared chemically and attached by photoactivation onto the PS surface. The surfaces were characterized by X-ray photoelectron spectroscopy (XPS) and time of flight secondary ion mass spectrometry (ToF-SIMS). In addition to hybridization experiments using rat hepatocytes and Allo A lectin, an enzymatic solid-phase glycosylation reaction of the lactose modified PS surface was investigated. Using recombinant $\alpha 2,6$-sialyltransferase expressed in Pichia pastoris allowed the incorporation of radioactive Neu5Ac from $\left[{ }^{14} \mathrm{C}\right]$-labeled CMPNeu5Ac at linear conversion rates for up to $4 \mathrm{~h}$ reaction time. After $6 \mathrm{~h}$ incubation time, enzymatic sialylations gave up to $40 \%$ product formation.

\section{Detection of Enzymatic Transformations Using Lectins and Antibodies}

A limitation of using labeled substrate donors as described in the last section is the limited availability of such compounds. Biotinylation is tolerated for sialyltransferases but might not be tolerated by other enzymes. An alternative way of detecting glycan structures is through well-established characterization using lectins and carbohydrate-binding antibodies (Fig. 5). Such detection methods are again limited by the availability of suitable lectins, but have the advantage that lectins are often linkage specific, and thus acceptor and donor specificity as well as regio- and stereoselectivity of the glycosidic bond can be examined.

The binding of lectins and antibodies to glycan arrays can be monitored in different ways. An increasingly popular glycoarray platform displays carbohydrates on self-assembled monolayers on gold allowing for the detection of protein binding by surface plasmon resonance (SPR). Houseman and Mrksich (1999) have applied SPR to characterize the binding of two lectins specific for GlcNAc and LacNAc (B. simplicifolia lectin II, E. cristagalli lectin) to determine glycosylation efficiencies of bovine $\beta 1,4$-galactosyltransferase I. SPR has allowed them to obtain quantitative binding data, and they have described experiments using different enzyme concentrations, ligand densities on the array, and kinetic studies. Clé 


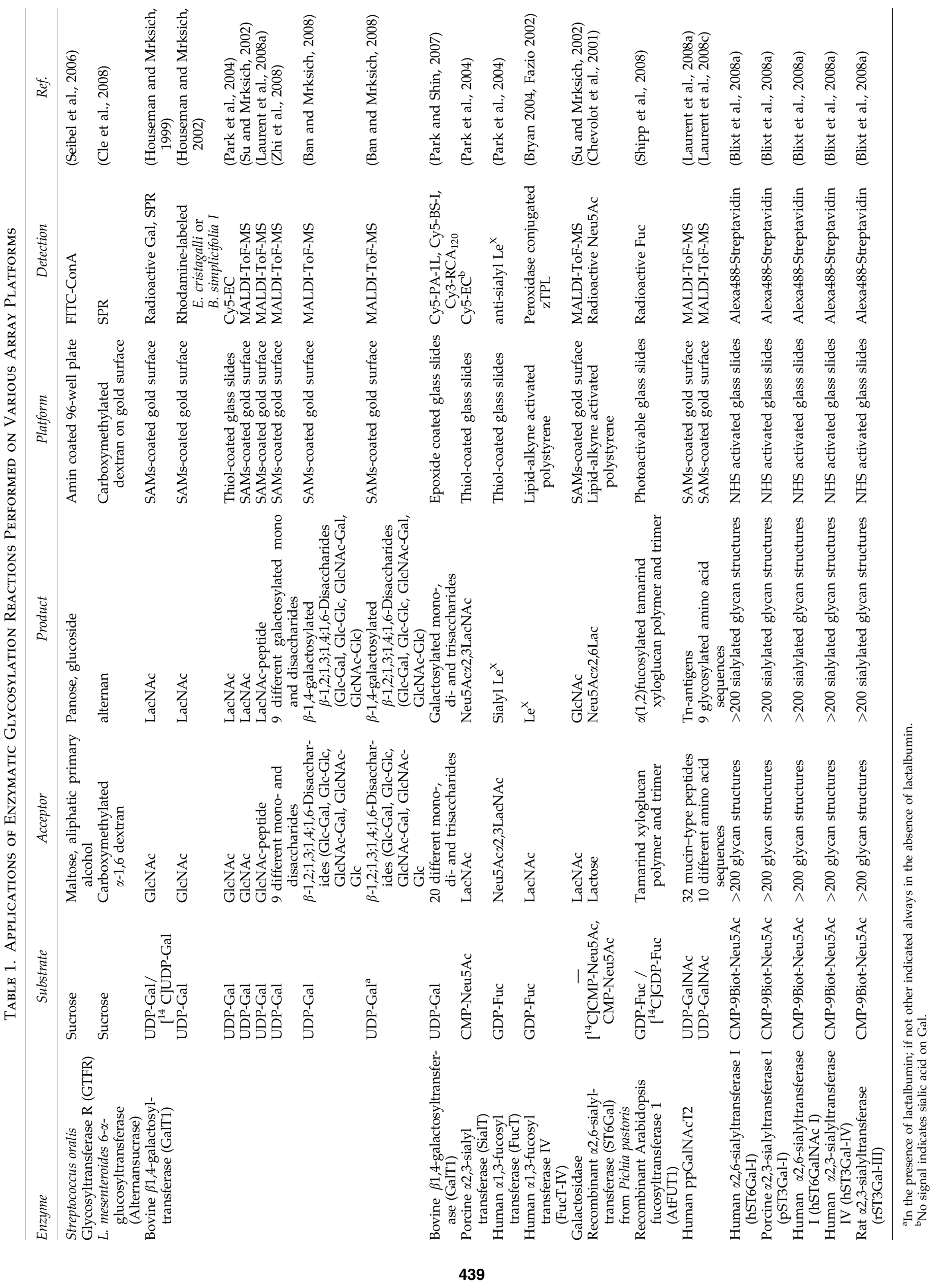




\section{Glycosyltransferase}
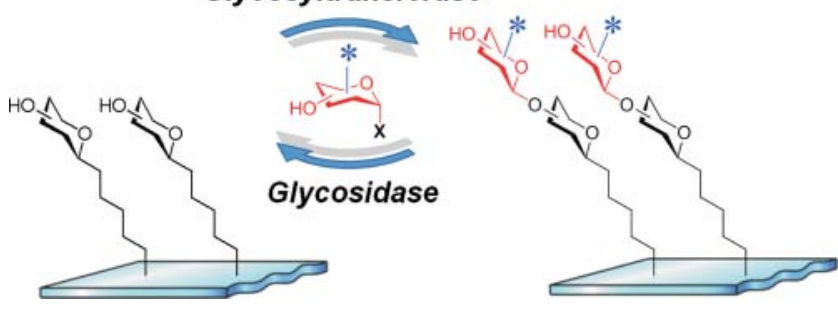

FIG. 2. Detection of enzyme activity on arrays using labelled glycosyl donors.

et al. (2008) have demonstrated the enzymatic transfer of glucose units to carboxymethylated dextran immobilized on gold arrays using real time monitoring by SPR spectroscopy in a label-free manner. Glucose was attached onto the immobilized carbohydrates using an $\alpha 1,6$-glucosyltransferase from Leuconostoc mesenteroides and sucrose as donor substrate. Besides the determination of the $k_{\mathrm{M}}$ value of the enzyme for sucrose, a linear relationship between increase of glucose transfer and enzyme concentration was demonstrated. Furthermore, the turnover numbers of the enzyme on the surface were estimated by the rate of polymer synthesis.

The bovine $\beta 1$,4-galactosyltransferase I (GalT1) has been a popular enzyme for proof-of-concept studies on different platforms, and there has generally been good agreements between methods with respect to acceptor specificities (Houseman and Mrksich, 2002; Zhi et al., 2008). The product can be detected using fluorescently labeled lectins even on gold surfaces, where fluorescence quenching can reduce sensitivity (Houseman and Mrksich, 2002; Zhi er al., 2006). Park and Shin (2007) have developed a glycoarray platform for the screening of $\beta 1$,4-galactosyltransferase on epoxidecoated glass slides. They attached 20 different mono-, di-, and trisaccharides to the glass surface, performed an enzymatic reaction and detected the product with a fluorescence marked lectin assay (Park and Shin, 2007). GalT1 showed higher activity with $\beta$-GlcNAc than $\alpha$-GlcNAc.

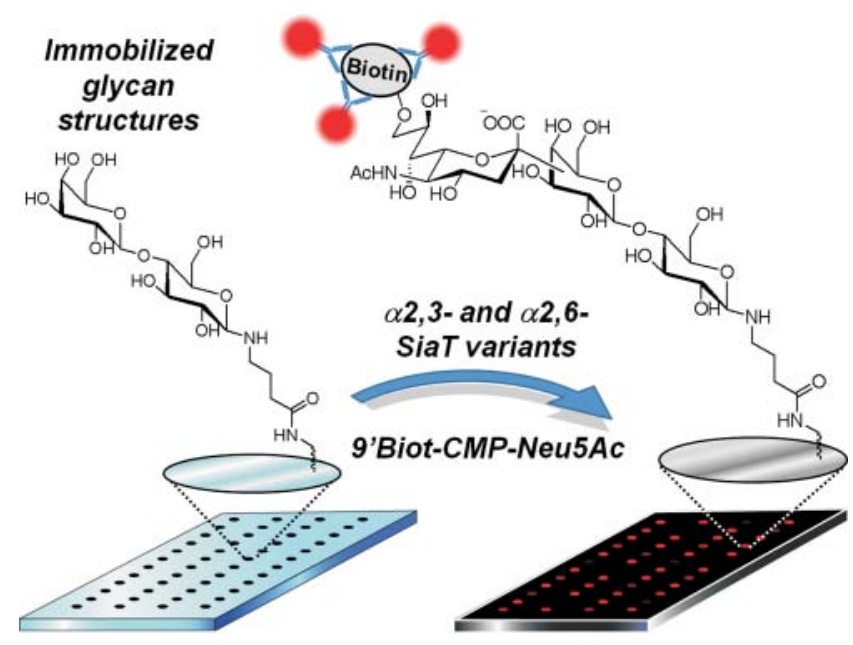

FIG. 3. Glycoarrays are used to monitor the substrate specificity of a range of alpha-sialyltransferases. The glycosyldonor is labeled as a biotin derivative (Blixt, 2006).

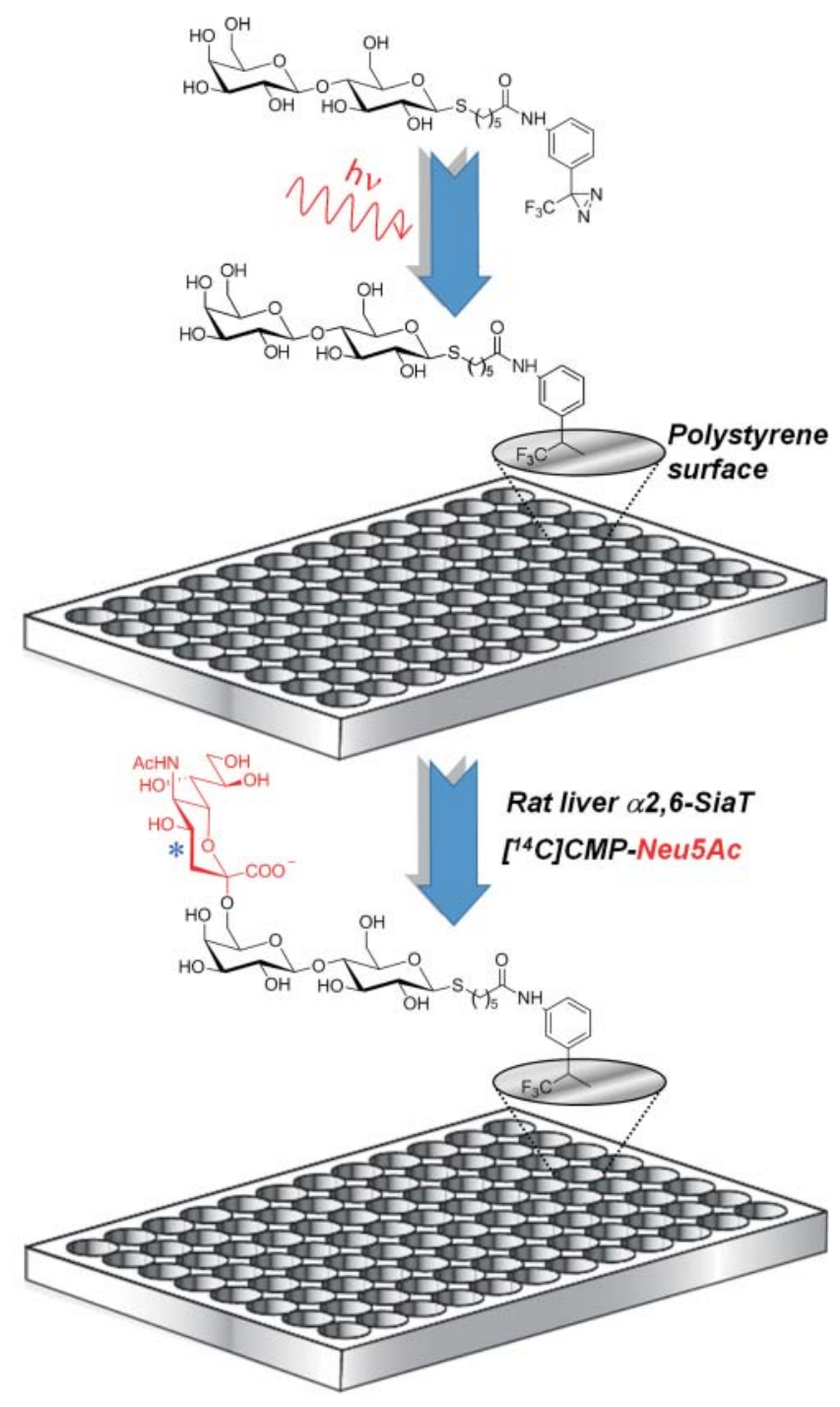

FIG. 4. Enzymatic modification of immobilized diazirine linked lactose by rat liver $\alpha 2,6$-sialyltransferase using radiolabelled CMP-Neu5Ac donor substrate (Chevolot, 2001).

The most complex glycan structure enzymatically synthesized on solid support so far is sialyl Le ${ }^{x}$ (Park et al., 2004; Shin et al., 2005). Park et al. synthesized this target by immobilizing maleimide-derivatized $\beta$-GlcNAc onto thiol-modified glass slides, followed by a series of consecutive enzymatic reactions using three recombinant glycosyltransferases (Fig. 6). To generate immobilized LacNAc, microspots containing immobilized GlcNAc where incubated with a reaction mixture containing bovine $\beta 1$,4-galactosyltransferase (GalT1) and UDP-galactose as a donor substrate. After rinsing of the solid support, this structure was further incubated with $\alpha 2,3$-sialyltransferase from porcine liver (SiaT) and CMP-Neu5Ac in an overnight reaction to generate immobilized Neu5Ac $\alpha 2,3-$ LacNAc. Finally, this spots were incubated with a human $\alpha 1,3$-fucosyltransferase (FucT) in the presence of GDP-Fuc, to generate the final sialyl $\mathrm{Le}^{x}$ tetrasaccharide immobilized on the chip. After each step the enzymatic elongation was monitored using fluorescence labelled lectins or antibodies specific for the generated glycan ( $\beta$-GlcNAc was probed with T. vul- 


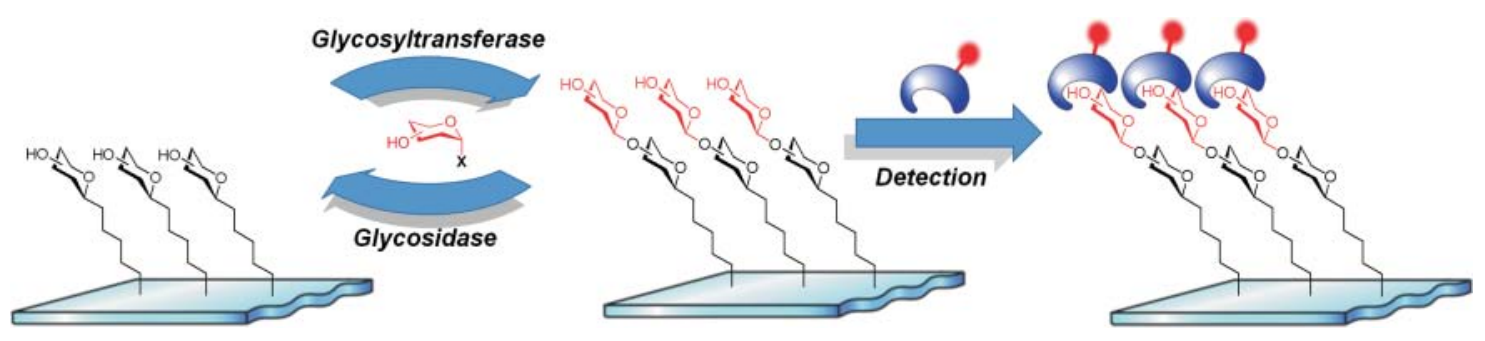

FIG. 5. Detection of enzymatic activity using labeled lectins or antibodies.

garis lectin (Cy3-TV), LacNAc with E. cristagalli lectin (Cy5EC), sialylation by loss of binding of Cy5-EC, and sialyl Le ${ }^{x}$ by using sequentially mouse anti-sialyl $\mathrm{Le}^{\mathrm{x}}$ - and goat Cy5-antiantibody). A similar approach to generate this structure on arrays was demonstrated by Fazio et al. (2002). In this case, the sialylated trisaccharide was immobilized and only the last fucosylation step was carried out directly on the array surface.

Seibel et al. (2006) demonstrated new acceptor specificities of the glycosyltransferase R (GTFR) from Streptococcus oralis (E.C. 2.4.1.5) using a microarray platform presenting immobilized carbohydrates and aliphatic alcohols as substrates. The covalent attachment of the compounds was performed by 1,3-dipolar cycloaddition of azidoalkyl glycosides with propyonic acid followed by NHS/EDC activation and coupling to amino-coated 96-well microtiter plates (Fig. 7) (Fazio et al., 2002). In contrast to other glycosyltransferases tested on microarray platforms so far (see Table 1), GTFR is a non-Leloirtype enzyme, and therefore utilizes nonactivated substrates like sucrose. This enzyme allows the direct transfer of a glucose unit from sucrose onto the immobilized acceptors. The glycosylation of acceptors was determined by measuring

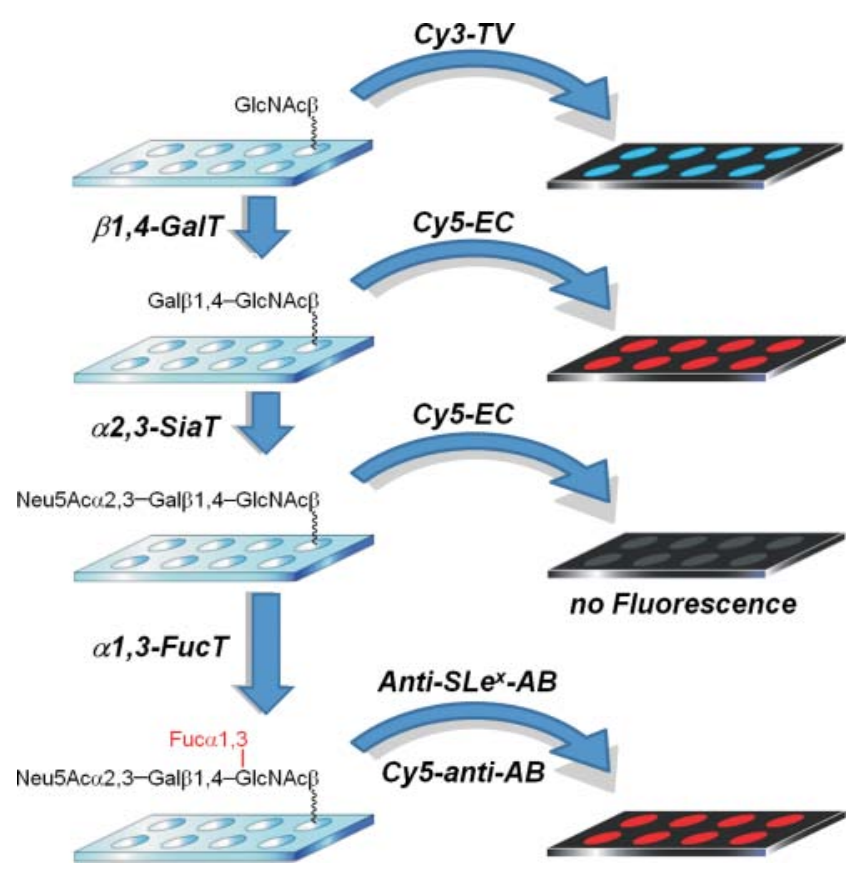

FIG. 6. Enzymatic generation of sialyl Le $\mathrm{e}^{\mathrm{x}}$ on solid support and detection of reaction products using antibodies and lectins (Shin, 2005). of fluorescence using the FITC-labeled mannose-/glucosespecific lectin Concanavalin A (ConA). For the first time, enzymatic glycosylation of a primary aliphatic alcohol been reported using microarray technology. The probing of the enzymatic glycosylation is based on an increase of the fluorescence signal for the product formed compared to the signal of the immobilized acceptors. When maltose, which is known to bind to ConA, was a starting material, an increase of fluorescence after the glycosylation reaction was observed. This is in agreement with earlier reports by Goldstein et al. (1965), who described a twofold increase of ConA binding specificity of the trisaccharide compared to maltose. In contrast, no glycosylation was observed on immobilized lactose. To identify the products further studies were performed in solution with maltose, various primary alcohols as well as serine and threonine derivatives.

Array technology has also been used as a tool for screening libraries of potential glycosyltransferase inhibitors. The group of Wong has studied the effect of known (Fazio et al., 2002) and novel (Bryan et al., 2004) inhibitors of a human $\alpha 1,3-$ fucosyltransferase IV (FucT-IV). All of the 86 tested compounds contained a guanosine diphosphate (GDP) moiety and a hydrophobic part, which should help to enhance the affinity toward a hydrophobic binding pocket close to the active site of the enzyme. The screening platform was based on lipidalkyne coated microtiter plates and LacNAc as acceptor substrate was immobilized via $\mathrm{Cu}(\mathrm{I})$-catalyzed 1,3-dipolar cycloaddition on the surface (Fig. 7). The enzymatic transfer of fucose from the donor substrate, GDP-Fuc onto the array platform was tested in the presence of potential inhibitors, and quantified using the fucose specific lectin Tetragonolobus purpureas (TPL) conjugated to a peroxidase. Four of the screened compounds showed inhibitory effects in the nanomolar concentration range.

\section{Label-Free Detection of Enzymatic Activity on Arrays}

The first studies involving enzymatic modifications on array platforms were reported a decade ago in the group of Mrksich (Houseman and Mrksich, 1999). These approaches were based on self-assembled monolayer-coated gold arrays in combination with SAMDI-ToF MS (self-assembled monolayers for matrix-assisted laser desorption-ionization time-offlight mass spectrometry) as detection systems (Ban and Mrksich, 2008; Houseman and Mrksich, 1999, 2002; Su and Mrksich, 2002). Combination of this array platform with the detection technique allows the analysis of immobilized glycan structures in a direct, label-free manner without the requirement for a lectin or antibody binding assays. In this methodology, arrays of 24 thioalkane derivatized disaccharides 

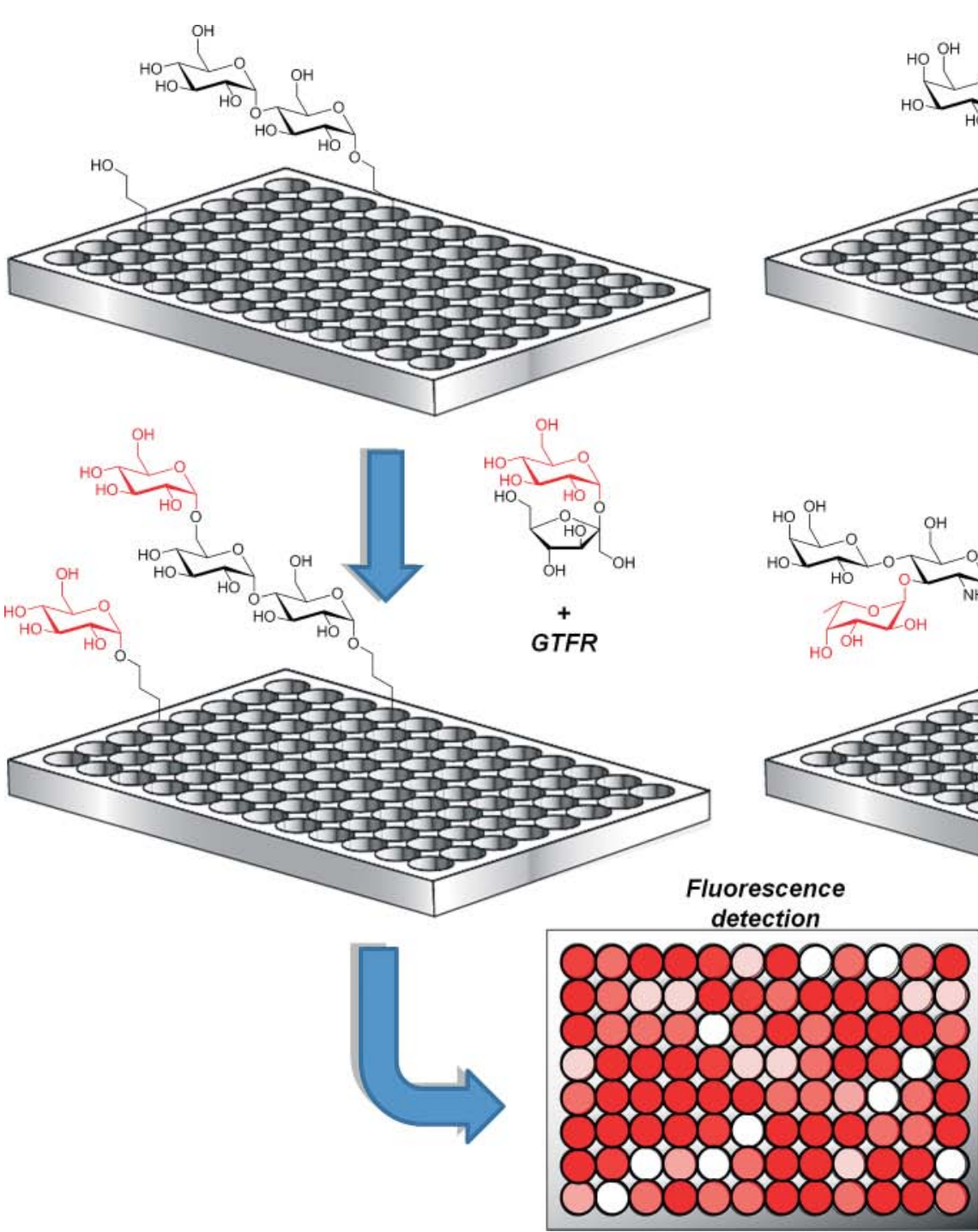

$\alpha 1,3-F u c T I V$

GDP-FUC

Inhibitor library

screening

FIG. 7. Left: glycosylation of covalently attached aliphatic alcohols and maltose with the enzyme GTFR (Glycosyltransferase R) (Seibel, 2006). Right: similar array system has been used to screen a panel of fucosyltransferase inhibitors (Bryan, 2004).

where immobilized via covalent attachment of the terminal thio group to a gold surface to screen the acceptor specificity of the bovine $\beta 1,4$-galactosyltransferase I. After addition of matrix (such as THAP, 2,4,6-trihydroxyacetophenone) the product can be directly measured by mass spectrometry without the need for cleavage (Schanbacher and Ebner, 1970). Approximate reaction yields have been determined by integration of MS peaks and comparing signal of starting material to that of product.

A method for screening of glycosyltransferases involved in protein $\mathrm{O}$-glycosylation of human mucins was developed by Laurent et al. (2008c) using the gold/SAM platform technology (Houseman et al., 2003; Houseman and Mrksich, 1999; Lamb et al., 2008; Petty et al., 2007; Qian et al., 2002; Yea et al., 2008; Zhi et al., 2006). A set of 10 peptides was immobilized in array format onto the SAM-coated gold surface, which was interrogated with isoform 2 of the human UDPGalNAc:polypeptide GalNAc-transferase family (ppGalNAcT). This family contains more than 20 human isoforms, all with distinct tissue specific and temporary expression pattern during development and also in adult tissue (Ten Hagen et al., 2003). However, in contrast to $N$-glycosylation, a consensus sequence for mucin-type glycosylation has not been found (Cai et al., 1997). The analysis by MALDI-ToF mass spectrometry could reveal different glycosylation efficiencies of different peptide substrates, and allowed the direct evaluation of the number of GalNAc residues added to peptides containing more than one potential glycosylation site (Fig. 8).

The gold platform is sufficiently robust to tolerate multistep chemical synthesis and allowed the combination of solidsupported peptide synthesis with enzymatic glycosylation methods. Peptide synthesis in array format are useful where a large number of peptide sequences need to be investigated. Based on the principle of SPOT synthesis (Frank, 1992), all chemical steps required for the peptide synthesis, such as repetitive cycles of amino acid couplings, $N$-Fmoc deprotection, acetylation, and TFA-mediated cleavage of protecting groups could be performed in situ (Fig. 8) (Laurent et al., 2008a). A series of peptides derived from the mucin Muc1 fragment AHGVTSAPA (with threonine as glycosyl-acceptor), was 


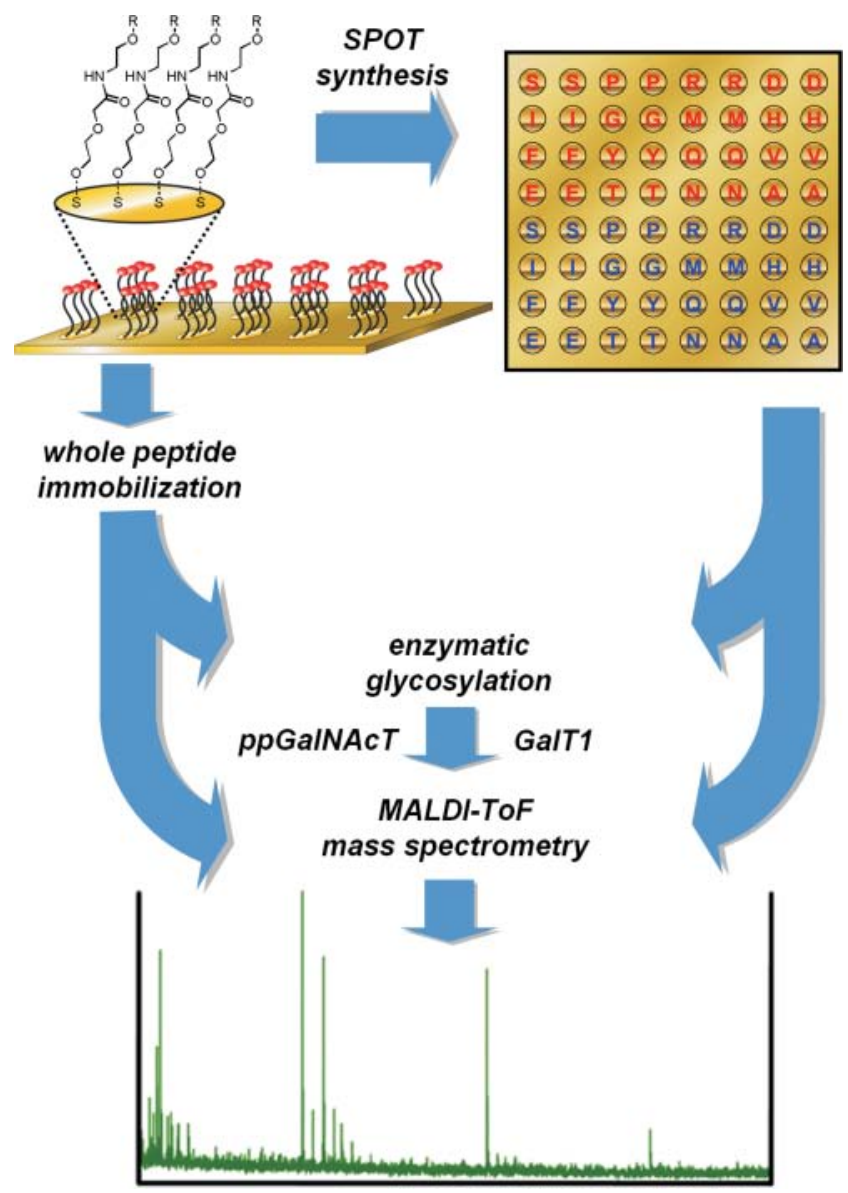

FIG. 8. Glycosylation of on gold immobilized peptides with the enzymes ppGalNAcT2 and GalT1. Products were detected in a label free manner using in situ MALDI-ToF mass spectrometry (Laurent, 2008a, 2008c).

prepared directly on the array. The effect of sequence variations at positions +1 and -1 in relation to threonine on the enzyme activity of the glycosyltransferase ppGalNAcT2 could be determined in a quantitative manner by MALDI-ToF MS. It was found that modifications at the -1 position relative to the threonine had a more severe effect on the glycosylation capability of ppGalNAcT 2 than the +1 position, and that an introduction of proline close to the threonine enhanced the glycosylation efficiency of the enzyme.

\section{Conclusion}

The application of array technology to the study of enzymes involved in glycosylation is a relatively young field and so far most reports have concentrated on method development. A range of platforms have been adapted from other array technologies (e.g., protein and nucleic acids) and so far have generated activity data that are in agreement with each other and with solution studies, although the data are still very limited. Given the advantages arrays bring to the investigation of glycoenzymes in terms of miniaturization and throughput, it is expected that glycoarrays will become important tools for glycoscientists. Because the glycoarray technologies use similar immobilization techniques and platforms as used for nucleic acid and peptide/proteins arrays, it will be possible to integrate glycans in multimodal analyses tools in future to study the intricate details of posttranslational modification.

\section{Acknowledgments}

The authors thank EPSRC and BBSRC (PhD Plus scheme for J.V.), the European Commission (Marie Curie IntraEuropean Fellowship for R.S and M.J.W), and the Royal Society (Wolfson Merit Award to S.L.F.) for financial support.

\section{Author Disclosure Statement}

The authors declare that no conflicting financial interests exist.

\section{References}

Ban, L., and Mrksich, M. (2008). On-chip synthesis and label-free assays of oligosaccharide arrays. Angew Chem Int Ed Engl 47, 3396-3399.

Blixt, O., and Razi, N. (2006). Chemoenzymatic synthesis of glycan libraries. Methods Enzymol 415, 137-153.

Blixt, O., Allin, K., Bohorov, O., Liu, X., Andersson-Sand, H., Hoffmann, J., et al. (2008a). Glycan microarrays for screening sialyltransferase specificities. Glycoconj J 25, 59-68.

Blixt, O., Hoffmann, J., Svenson, S., and Norberg, T. (2008b). Pathogen specific carbohydrate antigen microarrays: a chip for detection of Salmonella O-antigen specific antibodies. Glycoconj J 25, 27-36.

Bryan, M.C., Lee, L.V., and Wong, C.H. (2004). High-throughput identification of fucosyltransferase inhibitors using carbohydrate microarrays. Bioorg Med Chem Lett 14, 3185-3188.

Cai, Y.D., Yu, H., and Chou, K.C. (1997). Artificial neural network method for predicting the specificity of GalNActransferase. J Protein Chem 16, 689-700.

Cantarel, B.L., Coutinho, P.M., Rancurel, C., Bernard, T., Lombard, V., and Henrissat, B. (2009). The Carbohydrate-Active EnZymes database (CAZy): an expert resource for glycogenomics. Nucleic Acids Res 37(Database issue), D233-D238.

Chevolot, Y., Martins, J., Milosevic, N., Leonard, D., Zeng, S., Malissard, M., et al., (2001). Immobilisation on polystyrene of diazirine derivatives of mono- and disaccharides: biological activities of modified surfaces. Bioorg Med Chem 9, 2943-2953.

Cle, C., Gunning, A.P., Syson, K., Bowater, L., Field, R.A., and Bornemann, S. (2008). Detection of transglucosidase-catalyzed polysaccharide synthesis on a surface in real time using surface plasmon resonance spectroscopy. J Am Chem Soc 130, 15234-15235.

Davis, B.G. (2000). Recent developments in oligosaccharide synthesis. J Chem Soc Perkin Trans 1, 2137-2160.

Fazio, F., Bryan, M.C., Blixt, O., Paulson, J.C., and Wong, C.H. (2002). Synthesis of sugar arrays in microtiter plate. J Am Chem Soc 124, 14397-14402.

Frank, R. (1992). Spot-Synthesis—an easy technique for the positionally addressable, parallel chemical synthesis on a membrane support. Tetrahedron 48, 9217-9232.

Goldstein, I.J., Hollerman, C.E., and Smith, E.E. (1965). Proteincarbohydrate interaction. Ii. Inhibition studies on the interaction of concanavalin a with polysaccharides. Biochemistry 4 , 876-883.

Houseman, B.T., and Mrksich, M. (1999). The role of ligand density in the enzymatic glycosylation of carbohydrates presented on self-assembled monolayers of alkanethiolates on gold. Angew Chem Int Ed 38, 782-785. 
Houseman, B.T., and Mrksich, M. (2002). Carbohydrate arrays for the evaluation of protein binding and enzymatic modification. Chem Biol 9, 443-454.

Houseman, B.T., Gawalt, E.S., and Mrksich, M. (2003). Maleimide-functionalized self-assembled monolayers for the preparation of peptide and carbohydrate biochips. Langmuir $19,1522-1531$.

Lamb, B.M., Westcott, N.P., and Yousaf, M.N. (2008). Live-cell fluorescence microscopy of directed cell migration on partially etched electroactive SAM gold surfaces. Chembiochem 9, 2220-2224.

Laurent, N., Haddoub, R., Voglmeir, J., Wong, S.C., Gaskell, S.J., and Flitsch, S.L. (2008a). SPOT synthesis of peptide arrays on self-assembled monolayers and their evaluation as enzyme substrates. Chembiochem 9, 2592-2596.

Laurent, N., Voglmeir, J., and Flitsch, S.L. (2008b). Glycoarrays-tools for determining protein-carbohydrate interactions and glycoenzyme specificity. Chem Commun (Camb) 37, 4400-4412.

Laurent, N., Voglmeir, J., Wright, A., Blackburn, J., Pham, N.T., Wong, S.C., et al. (2008c). Enzymatic glycosylation of peptide arrays on gold surfaces. Chembiochem 9, 883-887.

Nicolaou, K.C., and Mitchell, H.J. (2001). Adventures in carbohydrate chemistry: new synthetic technologies, chemical synthesis, molecular design, and chemical biology. Angew Chem Int Ed Engl 40, 1576-1624.

Park, S., and Shin, I. (2007). Carbohydrate microarrays for assaying galactosyltransferase activity. Org Lett 9, 16751678.

Park, S., Lee, M.R., Pyo, S.J., and Shin, I. (2004). Carbohydrate chips for studying high-throughput carbohydrate-protein interactions. J Am Chem Soc 126, 4812-4819.

Park, S., Lee, M.R., and Shin, I. (2008). Carbohydrate microarrays as powerful tools in studies of carbohydrate-mediated biological processes. Chem Commun (Camb) 37, 4389-4399.

Petty, R.T., Li, H.W., Maduram, J.H., Ismagilov, R., and Mrksich, M. (2007). Attachment of cells to islands presenting gradients of adhesion ligands. J Am Chem Soc 129, 29, 8966.

Qian, X.P., Metallo, S.J., Choi, I.S., Wu, H.K., Liang, M.N., and Whitesides, G.M. (2002). Arrays of self-assembled monolayers for studying inhibition of bacterial adhesion. Anal Chem 74, 1805-1810.

Schanbacher, F.L., and Ebner, K.E. (1970). Galactosyltransferase acceptor specificity of the lactose synthetase A protein. J Biol Chem 245, 5057-5061.
Seeberger, P.H., and Haase, W.C. (2000). Solid-phase oligosaccharide synthesis and combinatorial carbohydrate libraries. Chem Rev 100, 4349-4394.

Seibel, J., Hellmuth, H., Hofer, B., Kicinska, A.M., and Schmalbruch, B. (2006). Identification of new acceptor specificities of glycosyltransferase $\mathrm{R}$ with the aid of substrate microarrays. Chembiochem 7, 310-320.

Shin, I., Park, S., and Lee, M.R. (2005). Carbohydrate microarrays: an advanced technology for functional studies of glycans. Chemistry 11, 2894-2901.

Shipp, M., Nadella, R., Gao, H., Farkas, V., Sigrist, H., and Faik, A. (2008). Glyco-array technology for efficient monitoring of plant cell wall glycosyltransferase activities. Glycoconj J 25, 49-58.

$\mathrm{Su}$, J., and Mrksich, M. (2002). Using mass spectrometry to characterize self-assembled monolayers presenting peptides, proteins, and carbohydrates. Angew Chem Int Ed Engl 41, 4715-4718.

Ten Hagen, K.G., Fritz, T.A., and Tabak, L.A. (2003). All in the family: the UDP-GalNAc: polypeptide N-acetylgalactosaminyltransferases. Glycobiology 13, 1R-16R.

Willats, W.G., Rasmussen, S.E., Kristensen, T., Mikkelsen, J.D., and Knox, J.P. (2002). Sugar-coated microarrays: a novel slide surface for the high-throughput analysis of glycans. Proteomics 2, 1666-1671.

Yea, C.H., Lee, B., Kim, H., Kim, S.U., El-Said, W.A., Min, J., et al. (2008). The immobilization of animal cells using the cysteinemodified RGD oligopeptide. Ultramicroscopy 108, 1144-1147.

Zhang, Z., Ollmann, I.R., Ye, X.-S., Wischnat, R., Baasov, T., and Wong, C.-H. (1999). Programmable one-pot oligosaccharide synthesis. J Am Chem Soc 121, 734-753.

Zhi, Z.L., Powell, A.K., and Turnbull, J.E. (2006). Fabrication of carbohydrate microarrays on gold surfaces: direct attachment of nonderivatized oligosaccharides to hydrazide-modified self-assembled monolayers. Anal Chem 78, 4786-4793.

Zhi, Z.L., Laurent, N., Powell, A.K., Karamanska, R., Fais, M., Voglmeir, J., et al. (2008). A versatile gold surface approach for fabrication and interrogation of glycoarrays. Chembiochem 9, 1568-1575.

Address correspondence to: Prof. Sabine L. Flitsch Manchester Interdisciplinary Biocentre University of Manchester Manchester M1 7ND, United Kingdom E-mail: sabine.flitsch@manchester.ac.uk 